\title{
Transvaginal artificial ascites infusion as a spacer in gynecological brachytherapy: a novel technique
}

\author{
Masataka Karube, MD, PhD!, Naoya Murakami, MD, PhD!' Hiroyuki Okamoto, PhD², Kae Okuma, MD, PhD!, \\ Tairo Kashihara, MD, PhD!, Kana Takahashi, MD, PhD!', Tomoya Kaneda, MD!, Koji Inaba, MD, PhD!, \\ Hiroshi lgaki, MD, PhD', Tomoyasu Kato, MD, PhD³, Jun Itami, MD, PhD' \\ 'Department of Radiation Oncology, National Cancer Center Hospital, Tokyo, Japan, ${ }^{2}$ Department of Medical Physics, National Cancer \\ Center Hospital, Tokyo, Japan, ${ }^{3}$ Department of Gynecologic Oncology, National Cancer Center Hospital, Tokyo, Japan
}

\begin{abstract}
This is a first paper to report on artificial ascites infusion via vaginal wall for pelvic interstitial brachytherapy. Artificial ascites is commonly used for treating liver tumors, with radiofrequency ablation and percutaneous artificial ascites infusion through the abdominal wall for pelvic brachytherapy has been previously reported by our group. However, the trans-abdominal needle approach under ultrasound guidance is unreliable due to poor visualization resulting in fluid injection into the abdominal wall or mesenterium and the rate of successful artificial ascites infusion was low. Target tumor of the vaginal cuff brachytherapy is usually adjacent to the intestine, and transvaginal artificial ascites infusion under trans-rectal ultrasonography is considered as a rational and simpler method to create a space between target volume and organs at risk, such as intestines or sigmoid colon, by increased visualization of the needle compared to trans-abdominal approach. Here, we report a practical experience of transvaginal artificial ascites infusion.

Key words: artificial ascites, spacer, transvaginal infusion, gynecological brachytherapy.

\section{Purpose}

Artificial ascites is commonly used for the radiofrequency ablation (RFA) of hepatocellular carcinoma or liver metastasis locating in the periphery of liver, forming a space between target lesion and adjacent gut [1,2,3,4,5]. Inspired by such clinical applications of artificial ascites, our group previously has reported percutaneous artificial ascites injection to pelvic brachytherapy for the first time [6]. By this method, it was possible to create a space between gross tumor volume (GTV) and organs at risk (OARs) as well as to reduce the doses to OARs. However, trans-abdominal needle insertion under trans-abdominal ultrasonography guidance was not reliable, and the success rate was not high enough for daily clinical use because visualization of the tip of the needle in the abdominal cavity was unfavorable due to the presence of gas in the bowel. As such, there were cases where saline was only injected into the abdominal wall or mesenterium, not into the aimed peritoneal cavity.

Here, we describe a practical experience of novel transvaginal artificial ascites infusion (TVAAI) under trans-rectal ultrasonography (TRUS), which is an easier approach to distribute the artificial ascites precisely to the intended peritoneal cavity with better visualization than the trans-abdominal injection.

\section{Case presentation}

A 42-year-old female with a vaginal cuff recurrence was referred to our department for high-dose-rate interstitial brachytherapy. At the age of 35 , she underwent simple hysterectomy for a cervical intraepithelial neoplasia. At the age of 42, a tumor in vaginal cuff was discovered by abnormal vaginal bleeding, and biopsy revealed small cell carcinoma. A whole-body physical examination, magnetic resonance imaging (MRI), and positron emission tomography (PET) showed that the disease invaded the left paravaginal tissue without reaching the pelvic wall. The greatest size of tumor was $34 \mathrm{~mm}$, and there was no evidence of distant metastasis. The patient was initially treated with 4 courses of cisplatin $\left(80 \mathrm{mg} / \mathrm{m}^{2}\right)$ and etoposide $\left(100 \mathrm{mg} / \mathrm{m}^{2}\right)$. Consecutively, she received intensity-modulated radiation therapy with $45 \mathrm{~Gy}$ in 25 fractions to the whole pelvis, and boost irradiation with $24 \mathrm{~Gy}$ in 4 fractions of high-dose-rate interstitial brachytherapy (HDR-ISBT), using remote after loading system. HDR-ISBT was performed once per day in four different days, with TVAAI performed in every session. PET-MRI after chemotherapy (before radiotherapy) showed a remaining activity of the vaginal cuff tumor (Figure 1). At the time of first brachytherapy, the size of tumor was reduced to $26 \mathrm{~mm}$. Figure 2 indicates 

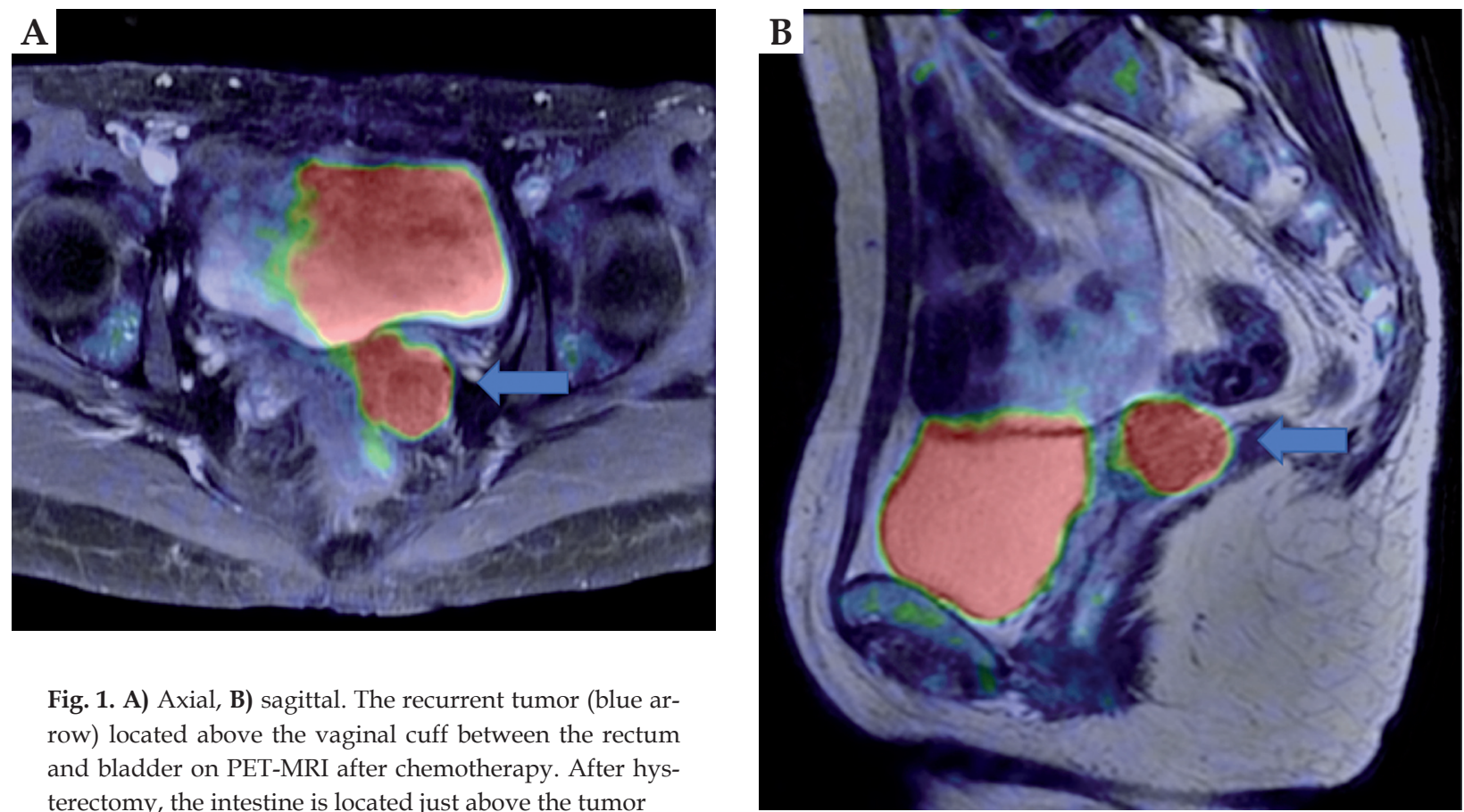

Fig. 1. A) Axial, B) sagittal. The recurrent tumor (blue arrow) located above the vaginal cuff between the rectum and bladder on PET-MRI after chemotherapy. After hysterectomy, the intestine is located just above the tumor

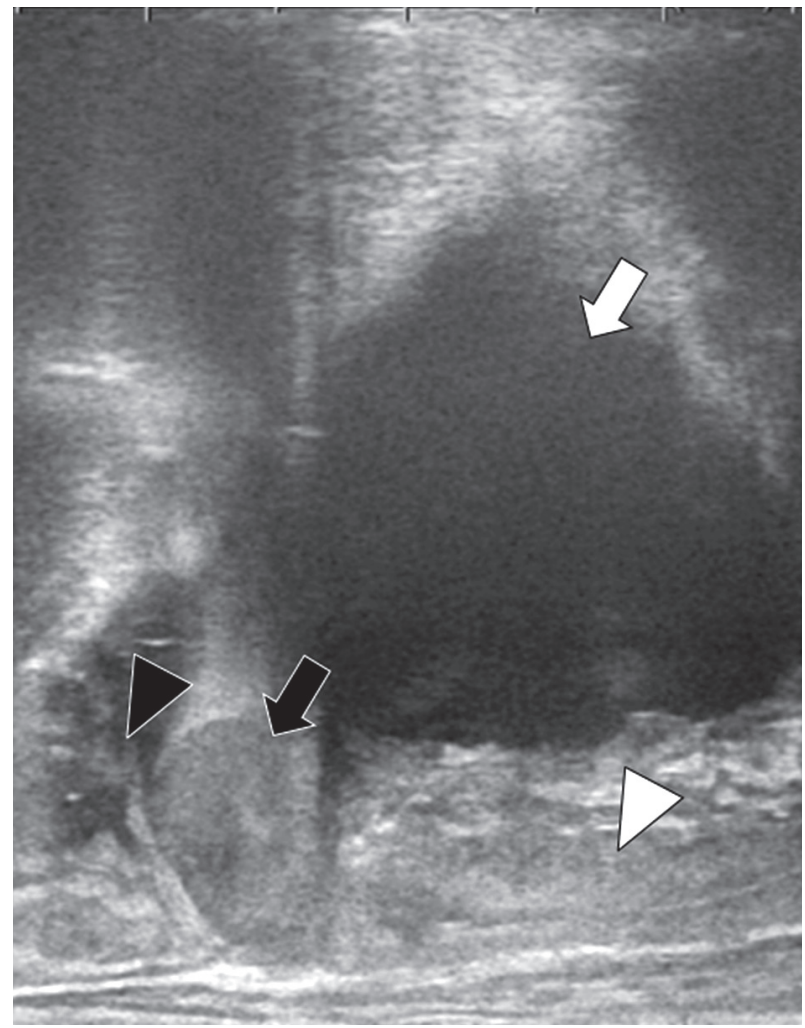

Fig. 2. Trans-rectal ultrasonography sagittal view before the first brachytherapy session. The recurrent tumor cranial to the vaginal cuff is next to the intestine. White arrow indicates the bladder, white arrowhead the vagina, black arrow the recurrent tumor, and black arrowhead the intestine, respectively the anatomical relationship of recurrent tumor and surrounding normal tissues in sagittal view of TRUS at the time of first brachytherapy.

\section{Transvaginal artificial ascites infusion and interstitial brachytherapy}

Before TVAAI, we performed a hyaluronate gel injection (HGI) to the pararectal space and posterior paravaginal space according to a technique reported by Kishi et al. [7]. Hyaluronate gel was injected through vaginal cavity under real-time TRUS guidance $[8,9,10]$. After finishing HGI, the needle was further proceeded and penetrated the peritoneum through the pouch of Douglas, with a careful attention not to damage the intestine. Awareness in avoiding tumor puncture was also required in order not to induce iatrogenic implantation. After the operators confirmed that the tip of needle was in the abdominal cavity, a $500 \mathrm{ml}$ injection of $5 \%$ glucose solution combined with $10 \mathrm{ml}$ of contrast agent was performed, and it was shown that the liquid moved the intestine away (Figure 3). For TVAAI, no additional anesthesia or sedation was necessary. After finishing TVAAI, vaginal cylinder and interstitial plastic needle applicators into the tumor under TRUS guidance were inserted $[11,12,13,14,15]$. Subsequently, planning computed tomography (CT) was taken with a slice thickness of $2 \mathrm{~mm}$ generated by a large bore CT simulator (Aquilion, Canon, Tokyo, Japan) with patients in lithotomy position. Finally, brachytherapy dose optimization was performed to apply the prescribed dose to the GTV with a maximal sparing of OARs, using Oncentra brachytherapy planning software (Elekta, Veenendaal, The Netherlands). 


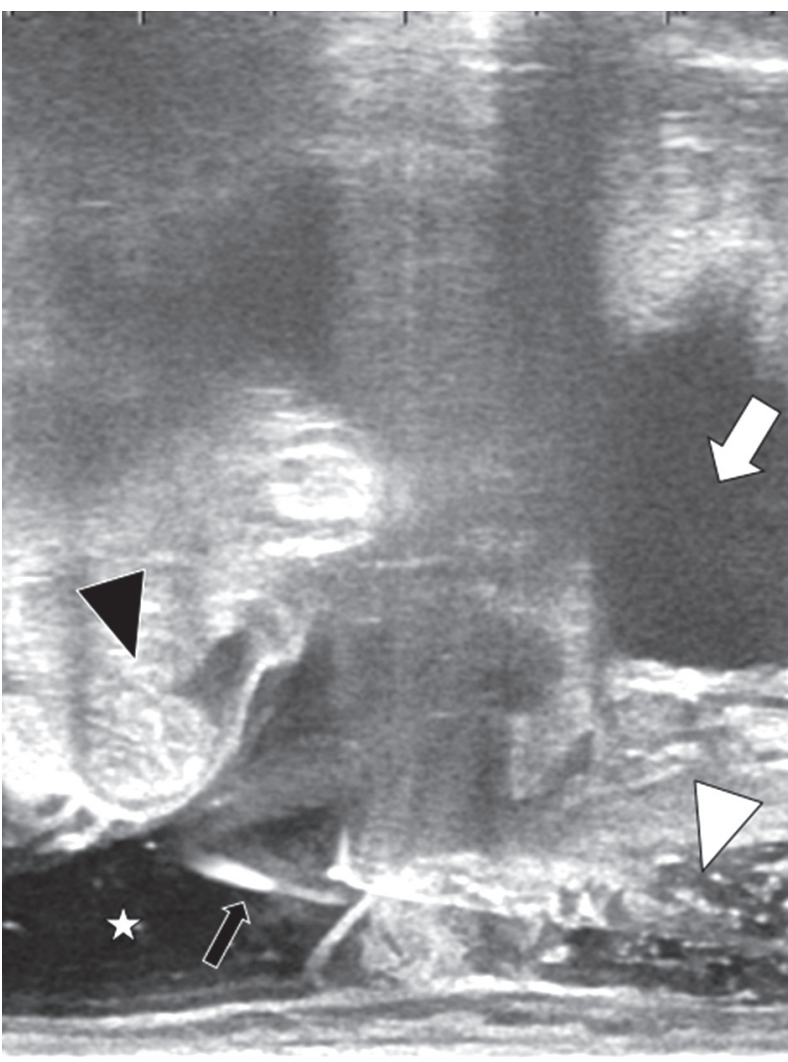

Fig. 3. Trans-rectal ultrasonography sagittal view during the injection of $5 \%$ glucose to abdominal cavity through the pouch of Douglas. The recurrent tumor is invisible in this section. The intestine was pushed away by the artificial ascites from the vaginal cuff. White arrow indicates the bladder, black arrowhead the intestine, white arrowhead hyaluronate gel between the vagina and rectum, and black arrow the needle penetrating the peritoneum, respectively. White asterisk shows artificial ascites in the abdominal cavity

Figure 4 displays the positions of all applicators in relation to the GTV and OARs. Normal tissue single-fraction dose before and after TVAAI, which was calculated at the first session of HDR-ISBT is summarized in Table 1. With the introduction of artificial ascites, intestinal $\mathrm{D}_{2 \mathrm{cc}}$ and sigmoid colon $\mathrm{D}_{2 \mathrm{cc}}$ was reduced from 350 cGy/229 cGy to $0 \mathrm{cGy} / 84 \mathrm{cGy}$, respectively. On the other hand, the rectum and bladder dose did not differ significantly before and after TVAAI. TVAAI was performed four times in every HDR-ISBT session, with all applications resulting in a success.

\section{Discussion}

Compared with percutaneous trans-abdominal injection of artificial ascites, TVAAI enables to infuse artificial ascites with better visualization of the needle tip as well as the injected fluid itself into targeted area. Additionally, HGI and TVAAI can be performed effortlessly, with the same needle without additional anesthesia or puncture.

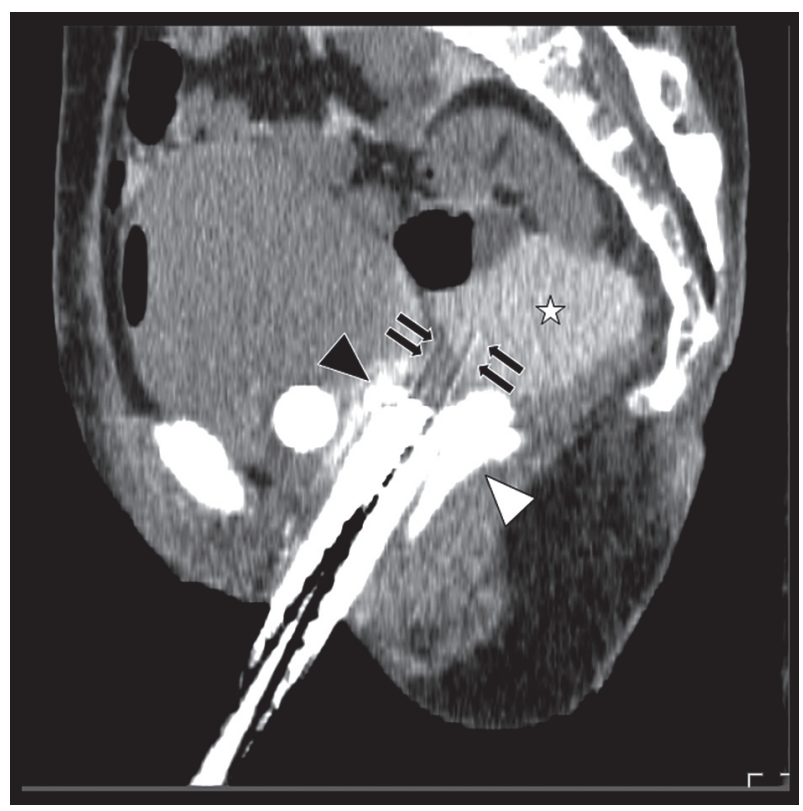

Fig. 4. Sagittal image of the planning CT after the procedures. White asterisk shows artificial ascites as $5 \%$ glucose with contrast enhancement agent, which lifted the intestine up from the tumor. Black and white arrowheads indicate hyaluronate gel spacing between the bladder and rectum, and black arrows show plastic interstitial brachytherapy needles penetrating the tumor. The artificial ascites and hyaluronate gel created distances between organ at risks and interstitial brachytherapy applicators

Table 1. Normal tissue single-fraction dose (first brachytherapy session) before and after transvaginal artificial ascites infusion (TVAAI)

\begin{tabular}{lcc} 
& Before TVAAl & After TVAAI \\
\hline Rectum $\mathrm{D}_{2 c c}(c G y)$ & 370 & 368 \\
\hline Bladder $\mathrm{D}_{2 c c}(c G y)$ & 419 & 418 \\
\hline Sigmoid colon $\mathrm{D}_{2 c c}(\mathrm{cGy})$ & 229 & 84 \\
\hline Intestine $\mathrm{D}_{2 c c}(c G y)$ & 350 & 0
\end{tabular}

There are spacer gels, which can be inserted into pararectal space to create an area between the rectum and prostate or vagina $[9,10,16]$. However, there are no agents, which can be injected into the peritoneal cavity, creating a space between vaginal stump of recurrent tumor and bowel adjacent to vaginal stump. TVAAI plays an important role as a spacer between the target volume and bowel floating in the peritoneal cavity using inexpensive materials. Although the intestinal dose could be $350 \mathrm{cGy}$ without the artificial ascites, TVAAI effectively reduced the dose to the intestinal while keeping the tumor dose sufficiently elevated. This technique is most appropriate in a re-irradiation setting, where surrounding intestines already receive certain doses of irradiation. 
The route of inserting a needle, in which no tumor exists between the puncture site and peritoneal cavity should be carefully planned beforehand, in order not to create iatrogenic implantation into the peritoneal cavity. If such a route exists, TVAAI can be applied for tumors with paravaginal invasion. So far, the actual rate of iatrogenic implantation related to TVAAI with needle penetrating tumor is not known and should be investigated in future researches. However, the authors believe that a dangerous needle pathway penetrating the tumor should be avoided when iatrogenic implantation is theoretically considered. TVAAI can also be applied for more common histology, such as squamous cell carcinoma or adenocarcinoma, as it happened in our case with small cell carcinoma.

The type of liquid, which should be used for artificial ascites for brachytherapy is not yet decided. In our previous report [6], we used saline. In contrast, when artificial ascites is used in RFA for liver tumors, $5 \%$ glucose solution is preferred because saline contains electrolytes, which provides a current. Moreover, the temperature of surrounding normal tissues around the treated site by RFA is higher with saline than that of $5 \%$ glucose solution, which does not passes the current [17]. Therefore, in brachytherapy, where unlike RFA, no high level of heat is created, both saline and $5 \%$ glucose solution are considered to be feasible. It is possible that saline would be preferred to $5 \%$ glucose solution in gynecologic brachytherapy to avoid infection where needles are inserted in the perineum, which includes the excretory organs. Especially for thin patients, if no contrast enhancement agent was used, it is difficult to distinguish artificial ascites from the bowel. Therefore, the authors recommend using contrast enhancement agent (if not contraindicated) to obtain better visualization.

As shown in this case report, repeated TVAAI is feasible with no severe complication related to this technique, supposedly because of no dangerous anatomic structures around the pouch of Douglas. However, because this novel technique is quite new, further experience with regard to toxicity is required.

There are several issues for TVAAI that should be considered. First, in case of tumor adhesion to bowel, even if artificial ascites is injected properly, no space between them is achievable. The presence of adhesion can be easily evaluated by TRUS: if the bowel next to the tumor moves smoothly, there is a doubtful presence of adhesion. Secondly, the time of fluid remaining in the peritoneal cavity is not known and should be investigated in further studies. Thirdly, complications or adverse effects related to TVAAI need to be additionally explored, including the rate of peritoneal dissemination, iatrogenic implantation, bowel injury, bleeding, or infections.

In conclusion, this report presented the easiness and effectiveness of TVAAI, which introduces an artificial ascites to the peritoneal cavity through the pouch of Douglas. This methodology was useful to create a space between the tumor target and OARs, such as the intestine, especially in the vaginal stump brachytherapy after hysterectomy. Further research is needed to investigate how the incidence of late radiation adverse effects for gastrointestinal tracts can be reduced by TVAAI.

\section{Declarations}

Ethics approval and consent to participate were acquired. Moreover, a written informed consent from patients for artificial ascites injection and brachytherapy were obtained. This case report was approved by the Institutional Review Board of the National Cancer Center Hospital (approval number, 2017-091) according to the ethical standards of the Declaration of Helsinki.

A written informed consent from the patient for publication, including each clinical datum as images, case history, and other clinical data was acquired.

\section{Funding}

This work was financially supported in part by the Japan Agency for Medical Research and Development (AMED), and the National Cancer Center Research and Development Fund (26-A-18 and 26-A-28).

\section{Disclosure}

Dr. Inaba report grants from Elekta outside the submitted work. Dr. Igaki declare grants from HekaBio and receives personal fee from Itochu outside the submitted work. Dr. Nakayama receives personal fees from AstrazZeneca outside the submitted work. Dr. Itami receives personal fees from HekaBio and from kay J outside the submitted work. The rest of the authors report no conflict of interest.

\section{References}

1. Wang CC, Kao JH. Artificial ascites is feasible and effective for difficult-to-ablate hepatocellular carcinoma. Hepatol Int 2015; 9: 514-519.

2. Uehara T, Hirooka M, Ishida $K$ et al. Percutaneous ultrasound-guided radiofrequency ablation of hepatocellular carcinoma with artificially induced pleural effusion and ascites. J Gastroenterol 2007; 42: 306-311.

3. Rhim H, Lim HK, Kim YS, Choi D. Percutaneous radiofrequency ablation with artificial ascites for hepatocellular carcinoma in the hepatic dome: initial experience. AJR Am J Roentgenol 2008; 190: 91-98.

4. Nishimura M, Nouso K, Kariyama K et al. Safety and efficacy of radiofrequency ablation with artificial ascites for hepatocellular carcinoma. Acta Med Okayama 2012; 66: 279-284.

5. Hsieh YC, Limquiaco JL, Lin CC et al. Radiofrequency ablation following artificial ascites and pleural effusion creation may improve outcomes for hepatocellular carcinoma in high-risk locations. Abdom Radiol (NY) 2019; 44: 1141-1151.

6. Murakami N, Shima S, Okuma K et al. Artificial ascites for organs at risk sparing in intrapelvic brachytherapy: a case report of recurrent uterine cervical carcinoma adjacent to the bowel. BJR Case Rep 2019; 5: 20180067.

7. Kishi K, Mabuchi Y, Sonomura T et al. Eradicative brachytherapy with hyaluronate gel injection into pararectal space in treatment of bulky vaginal stump recurrence of uterine cancer. J Radiat Res 2012; 53: 601-607.

8. Murakami N, Shima S, Kashihara T et al. Hyaluronic gel injection into the vesicovaginal septum for high-dose-rate brachytherapy of uterine cervical cancer: an effective ap- 
proach for bladder dose reduction. J Contemp Brachytherapy 2019; 11: 1-7.

9. Murakami N, Nakamura S, Kashihara T et al. Hyaluronic acid gel injection in rectovaginal septum reduced incidence of rectal bleeding in brachytherapy for gynecological malignancies. Brachytherapy 2020; 19: 154-161.

10. Kashihara T, Murakami N, Tselis N et al. Hyaluronate gel injection for rectum dose reduction in gynecologic high-doserate brachytherapy: initial Japanese experience. J Radiat Res 2019; 60: 501-508.

11. Umezawa R, Murakami N, Nakamura S et al. Image-guided interstitial high-dose-rate brachytherapy for locally recurrent uterine cervical cancer: A single-institution study. Brachytherapy $2018 ; 17$ : 368-376.

12. Sekii S, Murakami N, Kato T et al. Outcomes of salvage highdose-rate brachytherapy with or without external beam radiotherapy for isolated vaginal recurrence of endometrial cancer. J Contemp Brachytherapy 2017; 9: 209-215.

13. Murakami N, Kobayashi K, Shima S et al. A hybrid technique of intracavitary and interstitial brachytherapy for locally advanced cervical cancer: initial outcomes of a single-institute experience. BMC Cancer 2019; 19: 221.

14. Murakami N, Kobayashi K, Kato T et al. The role of interstitial brachytherapy in the management of primary radiation therapy for uterine cervical cancer. J Contemp Brachytherapy 2016; 8: 391-398.

15. Murakami N, Kato T, Miyamoto Y et al. Salvage High-doserate interstitial brachytherapy for pelvic recurrent cervical carcinoma after hysterectomy. Anticancer Res 2016; 36: 2413-2421.

16. Mariados N, Sylvester J, Shah D et al. Hydrogel spacer prospective multicenter randomized controlled pivotal trial: dosimetric and clinical effects of perirectal spacer application in men undergoing prostate image guided intensity modulated radiation therapy. Int J Radiat Oncol Biol Phys 2015; 92: 971-977.

17. Seike M, Yamashita T, Himeno K et al. Effective artificial ascites for RFA : whether we should use saline or $5 \%$ glucose solution? J Microwave Surg 2007; 25: 99-103. 